\title{
Propagación clonal de guayabo en distintos medios de enraizamiento
}

Clonal propagation of guava in different means of rooting

De Luna-Jiménez A. ${ }^{\circledR}$, Luna-Ruíz J.J. ${ }^{1}$, Fuántos-Mendoza J. ${ }^{1}$ Martínez de Lara J. ${ }^{1}$

${ }^{1}$ Departamento de Ciencias Agrícolas, Centro de Ciencias Agropecuarias, Universidad Autónoma de Aguascalientes, km 3 de la carretera Jesús María-La Posta Zootécnica, apartado postal 2, Jesús María, CP: 20900, Tel. 9107400. Ext. 8115. Aguascalientes, México.

${ }^{凶}$ Autor para correspondencia: $\underline{\text { lunaji@yahoo.com }}$

Recibido: 13/09/2019

Aceptado: 15/05/2019

\section{RESUMEN}

Existen registros del cultivo de guayaba en Calvillo desde 1824; actualmente se dedican al cultivo 2500 productores y se cosechan 95 mil toneladas anuales lo que generan una importante derrama económica. Con el objetivo de evaluar el impacto de seis sustratos solos (100\%) y mezclados con arena en proporciones iguales (50\% y 50\%), en el enraizamiento de estacas, en el Centro de Ciencias Agropecuarias de la UAA, se realizó durante 2017-18, la investigación. Las estacas se obtuvieron de árboles elite del Banco de Germoplasma de la UAA, se utilizaron macetas de plástico de $29 \mathrm{~cm}$ de alto y $30 \mathrm{~cm}$ de ancho, las que se llenaron dejando $3 \mathrm{~cm}$ de borde para el riego, las estacas, se impregnaron en la base con el enraizador radix 10,000 ppm y se cubrieron con bolsa plástica para crear una cámara húmeda. Se tuvieron dentro de un invernadero, se colocó sobre el techo una malla media sombra para reducir la luz, se regaron 3 veces por semana. Se registró la temperatura y humedad relativa a las 8:00 am, 12:00 pm y 16:00 pm, durante los meses de junio a octubre, se calculó para cada hora el promedio mensual. A los cinco meses se evaluaron las variables: número total de raíces (NTR), número de raíces por estaca (NRE), porcentaje de estacas enraizadas (\%EE) y longitud de la raíz más grande ( $\mathrm{LR}+\mathrm{G} \mathrm{cm})$. Los tratamientos con el mayor NTR, NRE, \%EE y LR+G, fueron Fibra de coco (100\%) (24.4 raíces, 4.889 raíces por estaca, $98 \%$ y $10.1 \mathrm{~cm})$, aserrín de mezquite + arena $(50 \%+50 \%),(24.1$ raíces, 4.83 raíces por estaca, $97 \%, 2.8 \mathrm{~cm}$ ) y paja de cereales (100\%) (22.8 raíces, 4.56 raíces por estaca, $91 \%, 7.2$ $\mathrm{cm})$.

Palabras clave: sustrato, estaca, enraizamiento.

\begin{abstract}
There are records of the cultivation of guava in Calvillo since 1824; currently, 2,500 producers are engaged in cultivation and 95,000 tons are harvested annually, which generates a significant economic


spill. With the objective of evaluating the impact of six substrates alone (100\%) and mixed with sand in equal proportions (50\% and 50\%), in the rooting of stakes, in the Center of Agricultural Sciences of the UAA, it was carried out during 2017-18, the investigation. The stakes were obtained from elite trees of the Germplasm Bank of the UAA, plastic pots $29 \mathrm{~cm}$ high and $30 \mathrm{~cm}$ wide were used, which were filled leaving $3 \mathrm{~cm}$ edge for irrigation, the stakes were impregnated in the base with the radix 10,000 ppm root and were covered with plastic bag to create a wet chamber. They were placed inside a greenhouse, a half shade mesh was placed on the roof to reduce the light, and they were watered 3 times a week. The relative temperature and humidity were recorded at 8:00 am, 12:00 pm and 4:00 pm, during the months of June to October; the monthly average was calculated for each hour. At five months, the variables were evaluated: total number of roots (NTR), number of roots per stake (NRE), percentage of rooted stakes (\% EE) and length of the largest root $(\mathrm{LR}+\mathrm{G} \mathrm{cm})$. The treatments with the highest NTR, NRE, \% EE and $\mathrm{LR}+\mathrm{G}$, were Coconut Fiber (100\%) (24.4 roots, 4,889 roots per stake, $98 \%$ and $10.1 \mathrm{~cm}$ ), mesquite sawdust + sand $(50 \%+50 \%),(24.1$ roots, 4.83 roots per stake, $97 \%, 2.8 \mathrm{~cm})$ and cereal straw $(100 \%)$ (22.8 roots, 4.56 roots per stake, $91 \%, 7.2 \mathrm{~cm})$.

Keywords: substrate, stake, rooting.

\section{INTRODUCCIÓN}

El cultivo a nivel nacional ocupa una superficie de 20,899 hectáreas, localizadas en veinte estados, aunque los más importantes son Michoacán, con 9,613 hectáreas, Aguascalientes, con 6,687 hectáreas y Zacatecas con 3,343 hectáreas (Cortes, 2017).

Existen registros del cultivo de guayaba en Calvillo, Aguascalientes, México, desde 1824; actualmente se producen 95 mil toneladas anuales de esta fruta y se dedican al cultivo alrededor de 2500 productores que generan una importante derrama económica sin considerar los empleos en la industria, transporte y comercialización de la fruta. (SAGARPA, 2017).

El cultivo enfrenta diversos problemas como la ocurrencia de heladas y granizadas, en 1997 y 1998 años en los que Aguascalientes y Zacatecas redujeron su producción por efecto de las heladas ocurridas en 1997. Esa situación, hizo que Michoacán participara en 2000 con $38 \%$ del volumen de producción nacional de guayaba, mientras que Aguascalientes aportó el 37\% y
Zacatecas 21\% (De Luna, 2013), la falta de competitividad en el precio con respecto a otras regiones del país, el cambio de muchos productores al dejar la guayaba para dedicarse a la producción de nopal, hortalizas, forrajes, o simplemente el abandono de sus huertas.

La propagación por semilla ha originado una gran variabilidad genética en los árboles, creando la necesidad de la multiplicación vegetativa, que asegure conservar las características genéticas de la planta madre y la capacidad de transmitirla de generación en generación (Farrés et al., 2009).

La propagación por estaca ha sido poco usada en guayabo ya que se considera una especie difícil de enraizar (Hartman y Kester, 2001); sin embargo, para facilitar tal labor es necesario utilizar las auxinas, en especial el ácido indo butírico (Pennock y Maldonado, 1963; Tingwa y Abadi, 1968). Para ello, dentro de los aspectos a considerar para propagar y desarrollar exitosamente plantas en vivero, la selección y el manejo del sustrato a emplear es uno de los más importantes. La correcta selección del sustrato 
en donde enraizarán las estacas y crecerán las plantas, juega un papel fundamental dado que el desarrollo y mantenimiento de un vigoroso sistema radical es esencial para el crecimiento de plantas saludables (Bilderback, 1982). Por consiguiente se evaluaron las variables: número total de raíces (NTR), número de raíces por estaca (NRE), porcentaje de estacas enraizadas $(\% \mathrm{EE})$, longitud de la raíz más grande $(\mathrm{LR}+\mathrm{G}$, $\mathrm{cm})$. Por lo tanto en esta investigación se planteó el objetivo de evaluar el efecto de diferentes sustratos sobre el enraizamiento de estacas de guayabo, y seleccionar el mejor.

\section{MATERIALES Y MÉTODOS}

Durante 2017 y 2018, en invernadero del Centro de ciencias Agropecuarias de la Universidad Autónoma de Aguascalientes (UAA), se realizó la investigación para evaluar el impacto de seis medios de enraizamiento (peat moss, estiércol de bovino, composta, aserrín de mezquite, paja de cereales y fibra de coco), la paja de cereales y fibra de coco se molieron en molino de martillos para tener partículas pequeñas, los seis medios se cribaron para disponer de materiales uniformes y se les sometió a solarización por un mes, como medida fitosanitaria.

Se utilizaron solos (100\%) y combinados con arena de rio lavada en proporciones iguales (50\% y 50\%), para conocer la retención de humedad, de cada sustrato se tomaron muestras por triplicado de $1 \mathrm{~L}$, se extendieron sobre papel absorbente a la sombra con ventilación natural, hasta que secaron, enseguida se llenaron los recipientes nuevamente y se pesaron, destarando el peso del recipiente, obteniéndose el peso de sustrato seco (PSS, g), ahora, se les suministró agua abundante dejándolos drenar libremente y cuando dejaron de drenar se pesaron nuevamente para conocer el peso de sustrato húmedo (PSH, g), la diferencia en peso (PSH-PSS=PAR) fue el peso del agua retenida (Cuadro 1, Figura 1).

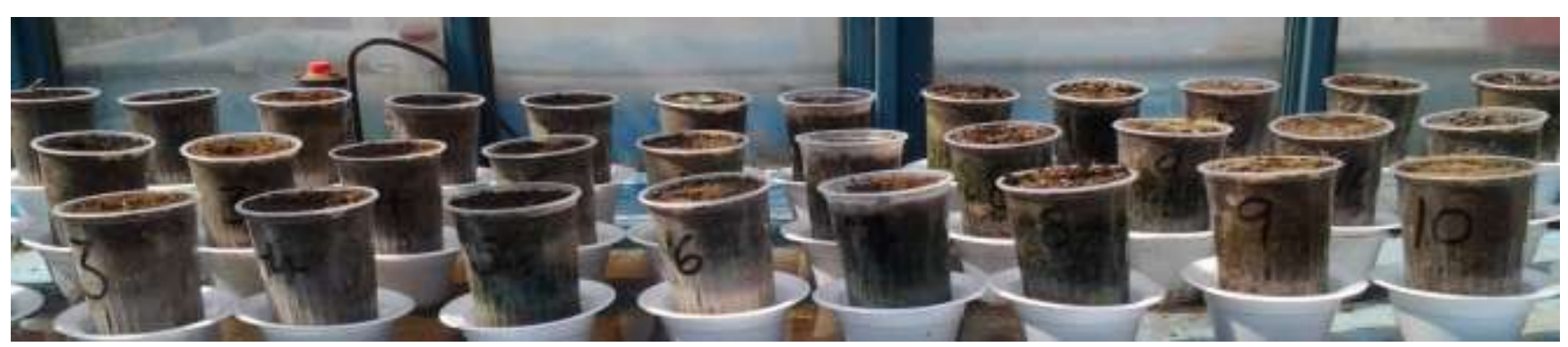

Figura 1. Determinación de la capacidad de retención de humedad de los medios experimentales (elaboración propia).

Con los medios solos y mezclados, se generaron 12 tratamientos y se usaron cuatro repeticiones, se utilizaron macetas de plástico de $29 \mathrm{~cm}$ de alto y $30 \mathrm{~cm}$ de ancho, las que se llenaron dejando 3 $\mathrm{cm}$ de borde para el riego, dando un total de 48 macetas, cada una fue una unidad experimental y se dispusieron en un arreglo completamente al azar.
Se cortaron ramas de árboles escogidos, se trasladaron al invernadero donde se obtuvieron las estacas entre 20 y $30 \mathrm{~cm}$ de longitud con cuatro nudos, las hojas basales se eliminaron, dejándoles únicamente el par de hojas de la punta, conforme se fueron obteniendo se sumergieron en un recipiente con agua $\mathrm{y}$ un fungicida (Figura 2). 


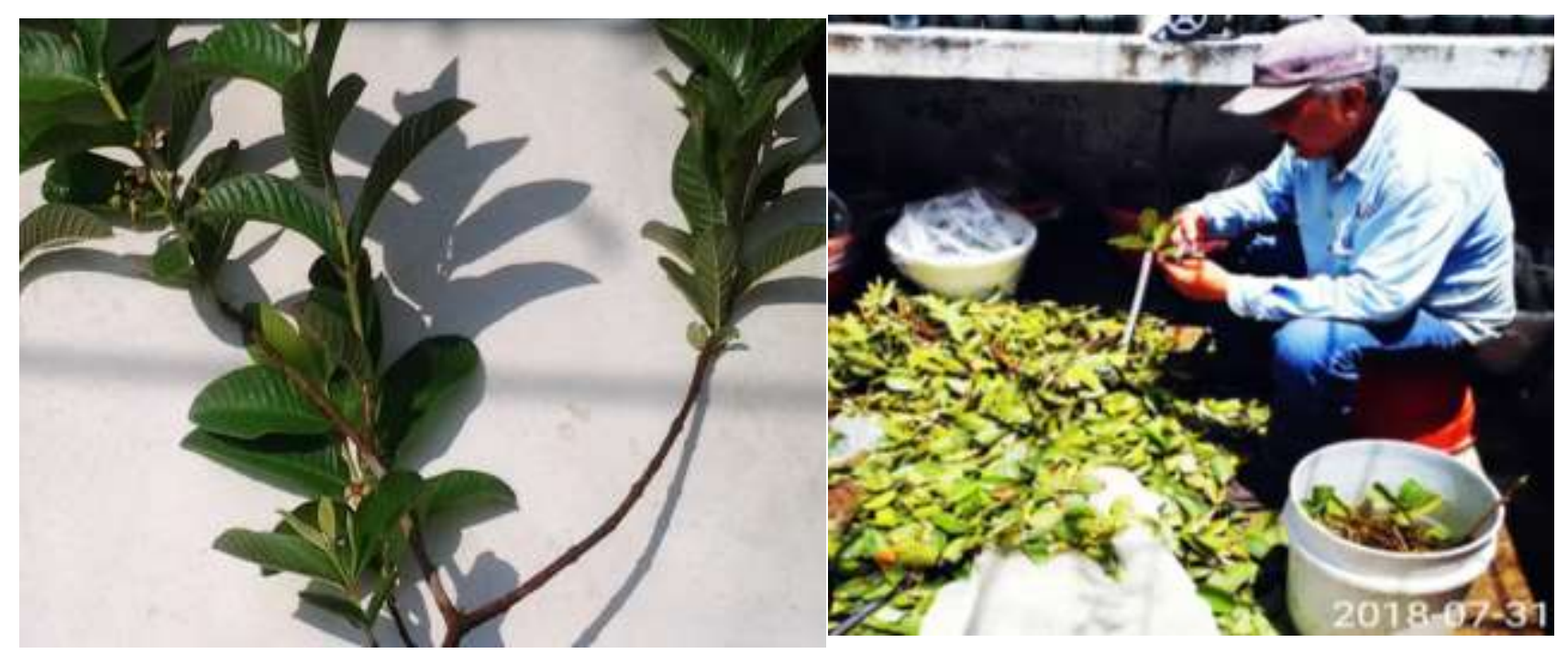

Figura 2. Rama, corte de estacas y desinfección (Fuente propia).

Por maceta se colocaron cinco estacas al momento de plantar, las estacas se impregnaron en la base con el enraizador radix 10,000 ppm, se clavaron cuatro soportes de madera de $40 \mathrm{~cm}$ de alto en cada maceta y se cubrieron con bolsa plástica transparente para crear una cámara húmeda, manteniendo en su interior un alto porcentaje de humedad relativa.

Se colocaron dentro de un invernadero, con ventilación natural y sobre el techo se colocó una malla sombra $30 \%$ monofilamento color negro para reducir la luz, se regaron 3 veces por semana y se tomaron muestras de los lixiviados para medir su pH y CE (Cuadro 2). Se llevó un registro diario de temperatura y humedad relativa a las 8:00 am, 12:00 pm y 16:00 pm, durante los meses de junio, julio, agosto septiembre y octubre, se calculó para cada hora el promedio mensual. A los cinco meses se evaluaron las variables: número total de raíces (NTR), número de raíces por estaca (NRE), porcentaje de estacas enraizadas (\%EE), longitud de la raíz más grande $(\mathrm{LR}+\mathrm{G}, \mathrm{cm})$. 
De Luna-Jiménez et al., 2019
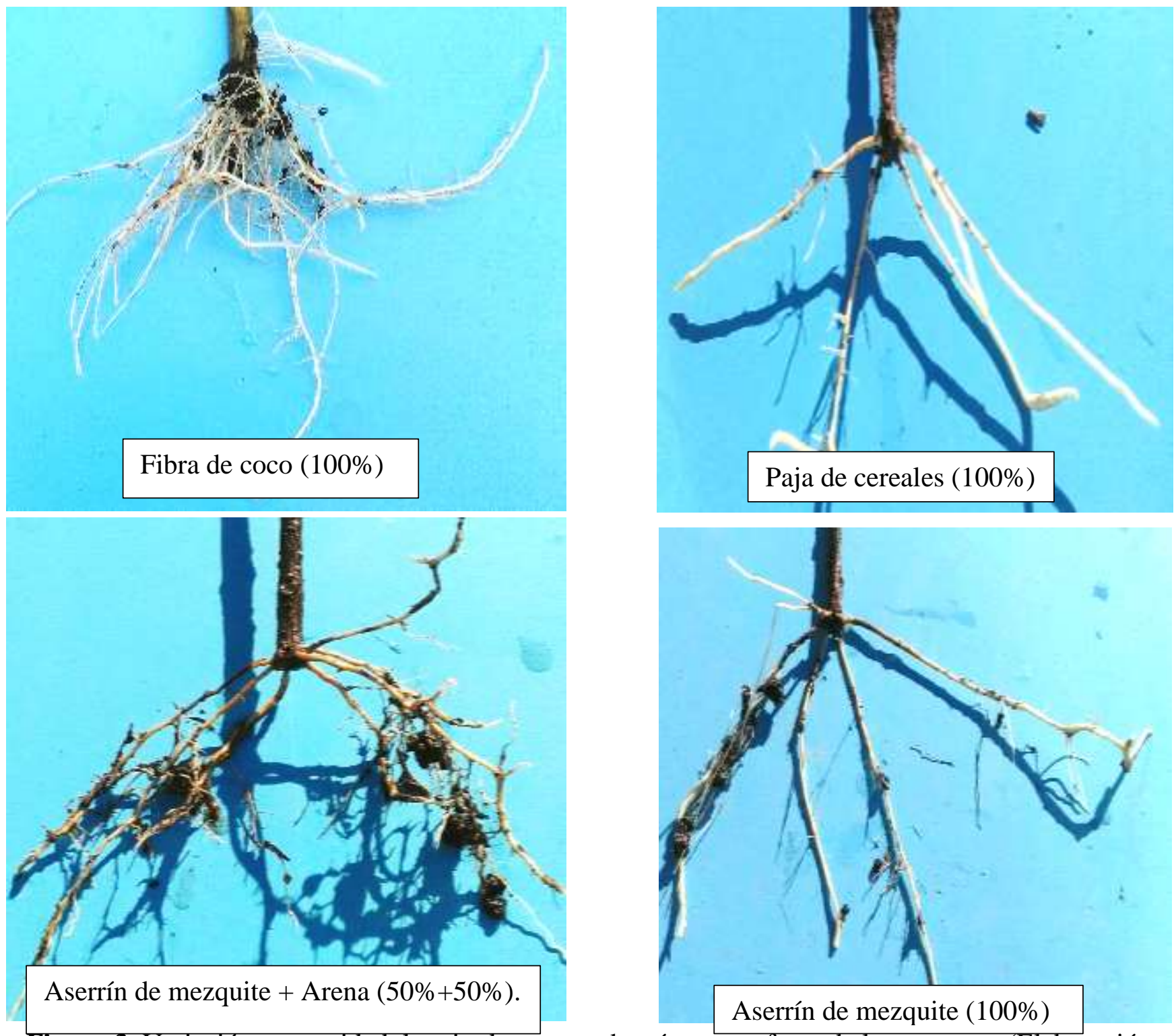

Figura 3. Variación en cantidad, longitud y grosor de raíces por efecto de los sustratos (Elaboración propia).

Los resultados se analizaron con el paquete estadístico Minitab 17, se realizó para cada variable el análisis de varianza y para la comparación de medias se utilizó la prueba de Tukey, 0.05 .

\section{RESULTADOS Y DISCUSIÓN}

Los sustratos $(100 \%)$, retuvieron más agua en comparación con los sustratos mezclados con arena $(50 \%+50 \%)$. El Peat moss $100 \%$, mostró la retención más alta $(313$ g) y el sustrato composta + arena $(50 \%+50 \%)$ la más baja (140 g) (Cuadro 1). La presencia de arena aunque redujo la retención, mejoró las condiciones físicas de los sustratos de acuerdo con Mello et al. (1983), Hartmann y Kester (2002) y Schmitz et al. (2002), quienes afirman que crea espacios porosos más grandes y aumenta la granulación en los sustratos, regulando la retención de agua y el drenaje favoreciendo el crecimiento de las raíces. 
Cuadro 1. Efecto de los sustratos en la retención de agua

\begin{tabular}{cccccccc}
\hline Sustrato100\% & PSS $(\mathrm{g})$ & PSH $(\mathrm{g})$ & PAR $(\mathrm{g})$ & Sustrato(50:50) & PSS $(\mathrm{g})$ & PSH $(\mathrm{g})$ & PAR $(\mathrm{g})$ \\
\hline Peat moss & $352 \mathrm{f}$ & $664 \mathrm{def}$ & 313 & Peat moss +Arena & $982 \mathrm{bc}$ & $1160 \mathrm{abc}$ & 178 \\
Aserrín & $428 \mathrm{f}$ & $714 \mathrm{de}$ & 286 & Aserrín + Arena & $905 \mathrm{~cd}$ & $1097 \mathrm{bc}$ & 192 \\
Composta & $847 \mathrm{~d}$ & $1037 \mathrm{c}$ & 190 & Composta + Arena & $1106 \mathrm{ab}$ & $1246 \mathrm{c} \mathrm{ab}$ & 140 \\
Estiércol & $615 \mathrm{e}$ & $781 \mathrm{~d}$ & 166 & Estiércol +Arena & $1124 \mathrm{a}$ & $1299 \mathrm{a}$ & 175 \\
Fibra de coco & $338 \mathrm{f}$ & $588 \mathrm{ef}$ & 250 & Fibra de coco+Arena & $952 \mathrm{~cd}$ & $1149 \mathrm{abc}$ & 197 \\
Paja & $356 \mathrm{f}$ & $549 \mathrm{f}$ & 193 & Paja + Arena & $880 \mathrm{~cd}$ & $1037 \mathrm{c}$ & 157 \\
\hline
\end{tabular}

PSS=Peso de sustrato seco (g), PSH= Peso de sustrato húmedo (g), PAR=Peso de agua retenida (g). Las medias que no comparten una letra son significativamente diferentes de acuerdo con la prueba de Tukey, 0.05 y una confianza de $95 \%$.

El pH medido en los lixiviados, en los tratamientos Peat moss (100\%), Peat moss + Arena $(50 \%+50 \%)$, Aserrín de mezquite $(100 \%)$, Aserrín de mezquite + Arena $(50 \%+50 \%)$, resultó ácido y en el resto de los tratamientos fue alcalino (Cuadro 2)
La conductividad eléctrica ( $\mathrm{CE} \mathrm{dSm}^{1}$ ), no mostró problema de salinidad, ya que entre los tratamientos, el rango fue de despreciable a muy ligeramente salino (Cuadro 2).

Cuadro 2. Variación del pH y conductividad eléctrica en los tratamientos

\begin{tabular}{lclcl}
\hline Tratamientos & $\mathrm{pH}$ & Interpretación & CEdSm $^{1}$ & Interpretación \\
\hline Peat moss $(100 \%)$ & 5.1 & Moderadamente Acido & 0.6 & Despreciable \\
Peat moss Arena $(50 \%+50 \%)$ & 5.4 & Moderadamente Acido & 1.0 & Despreciable \\
Aserrín de mezquite $(100 \%)$ & 5.0 & Fuertemente Acido & 0.9 & Despreciable \\
Aserrín de mezquite +Arena $(50 \%+50 \%)$ & 4.6 & Fuertemente Acido & 1.3 & Muy Lig. salino \\
Composta $(100 \%)$ & 7.8 & Medianamente alcalino & 1.7 & Muy Lig. salino \\
Composta+ Arena $(50 \%+50 \%)$ & 7.5 & Medianamente alcalino & 1.5 & Muy Lig. salino \\
Estiércol de bovino $(100 \%)$ & 7.8 & Medianamente alcalino & 1.6 & Muy Lig. salino \\
Estiércol de bovino + Arena $(50 \%+50 \%)$ & 7.4 & Medianamente alcalino & 1.9 & Muy Lig. salino \\
Fibra de coco $(100 \%)$ & 8.1 & Medianamente Alcalino & 0.7 & Despreciable \\
Fibra de coco + Arena $(50 \%+50 \%)$ & 7.8 & Medianamente alcalino & 0.9 & Despreciable \\
Paja de cereales $(100 \%)$ & 7.4 & Medianamente alcalino & 1.9 & Muy Lig. salino \\
Paja de cereales +Arena $(50 \%+50 \%)$ & 7.6 & Medianamente alcalino & 0.8 & Despreciable \\
\hline
\end{tabular}

El promedio mensual de temperatura fue más bajo a las 8:00 am; más alto a las 12:00 pm y se conservó alto hasta las 16:00 pm (Cuadro 3). Lo contrario se registró con $\mathrm{HR} \%$, a las 8:00 am fue alta y se redujo notablemente hasta las 12:00 pm, manteniéndose baja hasta las 16:00 pm (Cuadro $3)$. 
De Luna-Jiménez et al., 2019

Cuadro 3. Promedio mensual de temperatura $\left({ }^{\circ} \mathrm{C}\right)$ y humedad relativa $(\%)$

\begin{tabular}{ccccccc}
\hline & \multicolumn{2}{c}{$8: 00 \mathrm{am}$} & \multicolumn{2}{c}{$12: 00 \mathrm{pm}$} & \multicolumn{2}{c}{$16: 00 \mathrm{pm}$} \\
\hline Mes & $\mathrm{T}^{\circ} \mathrm{C}$ & $\mathrm{HR} \%$ & $\mathrm{~T}{ }^{\circ} \mathrm{C}$ & $\mathrm{HR} \%$ & $\mathrm{~T}{ }^{\circ} \mathrm{C}$ & $\mathrm{HR} \%$ \\
Junio & 18.9 & 82.7 & 33.6 & 41.4 & 32.0 & 41.4 \\
Julio & 22.4 & 55.8 & 23.2 & 33.3 & 23.0 & 56.6 \\
Agosto & 18.9 & 75.4 & 30.3 & 43.3 & 30.3 & 40.4 \\
Septiembre & 17.9 & 81.2 & 30.0 & 49.5 & 28.9 & 50.3 \\
Octubre & 23.7 & 55.2 & 23.4 & 56.5 & 23.9 & 54.2 \\
\hline
\end{tabular}

Cuadro 4. Efecto de los tratamientos en el enraizamiento de estacas

\begin{tabular}{lcccc}
\hline Tratamientos & NTR & NRE & \%EE & LR+G(cm) \\
\hline Peat moss $(100 \%)$ & 17.4 & $3.483 \mathrm{ab}$ & 70 & 2.9 \\
Peat moss Arena $(50 \%+50 \%)$ & 9.8 & $1.963 \mathrm{ab}$ & 39 & 2.5 \\
Aserrín de mezquite $(100 \%)$ & 18.9 & $3.778 \mathrm{ab}$ & 76 & 5.3 \\
Aserrín de mezquite +Arena $(50 \%+50 \%)$ & 24.1 & $4.83 \mathrm{a}$ & 97 & 2.8 \\
Composta $(100 \%)$ & 13.9 & $2.778 \mathrm{ab}$ & 56 & 4.7 \\
Composta+ Arena $(50 \%+50 \%)$ & 13.9 & $2.722 \mathrm{ab}$ & 54 & 3.1 \\
Estiércol de bovino $(100 \%)$ & 1.4 & $0.278 \mathrm{~b}$ & 6 & 0.3 \\
Estiércol de bovino + Arena $(50 \%+50 \%)$ & 15.3 & $3.056 \mathrm{ab}$ & 61 & 6.2 \\
Fibra de coco $(100 \%)$ & 24.4 & $4.889 \mathrm{a}$ & 98 & 10.1 \\
Fibra de coco + Arena $(50 \%+50 \%)$ & 16.6 & $3.33 \mathrm{ab}$ & 67 & 4.0 \\
Paja de cereales $(100 \%)$ & 22.8 & $4.56 \mathrm{a}$ & 91 & 7.2 \\
Paja de cereales +Arena $(50 \%+50 \%)$ & 11.2 & $2.239 \mathrm{ab}$ & 45 & 5.2 \\
\hline
\end{tabular}

NTR=Número total de raíces, NRE=Número de raíces por estaca, \%EE=Porcentaje de estacas enraizadas, LR+G $(\mathrm{cm})=$ Longitud de la raíz más grande. Promedios seguidos de la misma letra son estadísticamente iguales y con letras diferentes son distintos (Tukey, 0.05).

Los tratamientos que propiciaron el mayor número total de raíces (NTR), número de raíces por estaca (NRE) y porcentaje de estacas enraizadas (\%EE), fueron Fibra de coco $(100 \%)$, Aserrín de mezquite + arena $(50 \%+50 \%)$ y Paja de cereales $(100 \%)$, con el tratamiento Fibra de coco $(100 \%)$ se logró la raíz más grande (Cuadro 4).

\section{CONCLUSIONES}

Los medios de enraizamiento (sustratos) mostraron diferencias en su capacidad de enraizamiento y destacaron los tratamientos
Fibra de coco (100\%), Paja de cereales (100\%) y Aserrín de mezquite + Arena $(50 \%+50 \%)$. El mejor resulto Fibra de coco (100\%). En proyectos futuros de investigación o en producción de planta por viveristas se pueden considerar como promisorios.

\section{LITERATURA CITADA}

Bilderback, T. 1982. Nursey crops production manual. Container soils and soilless media. North Carolina Agricultural Extensión Service. NCPM. No. 9. 12 p. 
Cortés Solís, Judith. 2017. El sistema socioecológico de las huertas de guayaba en calvillo, Aguascalientes (Tesis de Maestría), Universidad autónoma de Aguascalientes. Aguascalientes, Ags. Recuperado de:http://bdigital.dgse.uaa.mx:8080/xmlui /handle/11317/1405? show=full

De Luna, A. (2013). Estudio de la guayaba. Aguascalientes, México. : UAA.

Farrés, E.; Placeres, G.J.: Rodríguez, D.A.; Peña, G.O. y Mulen, P. L.2009. Manual sobre la propagación de frutas tropicales. Instituto de Investigaciones en Fruticultura Tropical (IIFT). Ciudad de la Habana, Cuba.

Hartman, H. y D. Kester. 2001. Propagación de Plantas. Principios y prácticas. Octava reimpresión. Editorial Continental. México. 760 p.

HARTMANN， H.T.; KESTER， D.E. 2002. Plant propagation: principles and practices. 7. Ed. New Jersey: PrenticeHall, 880p.
MELLO, F.A.F.; SOBRINO, M.O.C.B.; ARZOLLA, S.; SILVEIRA, R.; NETTO, A.C.; KIEHL, J.C. 1983. Fertilidad do solo. São Paulo: Nobel. 400p.

Pennock, W.; Maldonado, G. 1963. The Propagation of guavas from stem cuttings. Journal of Agriculturere. Universidad de Puerto Rico. 47:280-290. https://doi.org/10.46429/jaupr.v47i4.1296 5

SAGARPA. (09 de marzo de 2017). SIAP. Obtenido de Servicio de Información Agroalimentaria y Pesquera.: http://infosiap.siap.gob.mx/index.php?opt ion=comwrapper\&view=wrapper \&Itemid $=240$

SCHMITZ, J.A.K.; SOUZA, P.V.D.; KÄMPF, A.N. 2002. Propriedades químicas e físicas de substratos de origen mineral e orgánico para o cultivo de mudas en recipientes. Ciencia Rural, Santa Maria, v.32, n.6, p.937-944. https://doi.org/10.1590/S0103-847820020 00600005

Tingwa, P. O.; Abadi, S. 1968. The vegetative propagation of the tropical guava (Psidium guajava L) in the Sudan. Agricultural Journal. 910:12-20. 
Copyright (c) 2019 A. De Luna-Jim énez, J. J. Luna-Ruiz, J. Fuántos-Mendoza, J. Martinez de Lara

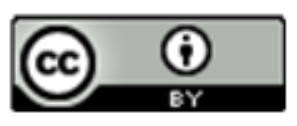

Este texto está protegido por una licencia licencia CreativeCommons 4.0.

Usted es libre para Compartir —copiar y redistribuir el $m$ aterial en cualquier medio o formato- y Adaptar el documento —remezclar, transformar y crear a partir del material- para cualquier propósito, incluso para fines com erciales, siempre que cumpla la condición de:

Atribución: Usted debe dar crédito a la obra original de manera adecuada, proporcionar un enlace a la licencia, e in dicar si se han realizado cambios. Puede hacerlo en cualquier forma razonable, pero no de forma tal que sugiera que tiene el apoyo del licenciante olo recibe por el uso que hace de la obra.

\section{Resumendelicencia $-\underline{\text { Textocompletodelalicencia }}$}

